\title{
Transmasculinización y hegemonía: opresión y género en las formas de ejercicio del poder político
}

\section{Transmasculinization and hegemony: gender and oppression in the different forms of exercise of political power}

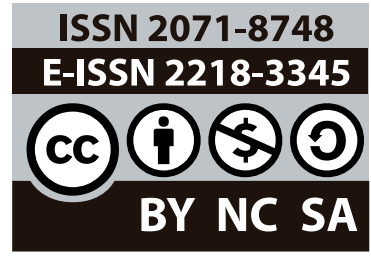

DOI: https://doi.org/10.5377/entorno.v0i69.9565 URI: http://hdl.handle.net/11298/1159

\author{
Astrid Yohanna Del Aguila de León \\ Licenciada en Relaciones Internacionales \\ Universidad de San Carlos de Guatemala \\ Guatemala, Centroamérica \\ Email: ayaldeleon@hotmail.es \\ ORCID: https://orcid.org/0000-0002-0500-3695
}

Recibido: 13 de enero 2020

Aceptado: 27 de junio de 2020

\section{Resumen}

La investigación teoriza sobre la forma del ejercicio del poder político donde el sujeto o actor social políticamente relevante, con independencia de su identidad de género, se ve condicionado por el entorno en el cual despliega sus relaciones sociales, presionando o constituyendo su identidad y devenir como sujeto sometido a esos mandatos sociales o políticos propios del poder que ostenta.

Cuando las mujeres logran acceder a un cargo de poder siguen siendo juzgadas por "patrones de asimilación" masculinos, $y$, al no encajar con ese modelo, llegan a ser discriminadas, cuestionadas o juzgadas en su papel político, observándose que la reacción de algunas mujeres en cargos de poder es adoptar formas y modelos masculinos hegemónicamente aceptados., por lo que la investigación da cuenta sobre la naturaleza de dicho fenómeno: este

\section{Abstract}

This study theorizes on the forms of exercising political power where the polittically of socially relevant actor, regardless of their gender identity-see themselves conditioned by the environment in which their social relations are performed, pushing or constituting their identity and evolution as an individual subjected to those social or political orders so characteristic of said type of power.

When women gain access to a position of power, they keep on being judged by masculine "assimilation patterns" and are discriminated, judged, or questioned in their political role since they do not fit that model. It has been observed that the reaction in some of the women in a power position is to adopt the masculine forms and models hegemonically accepted. Therefore, the research gives an account on the nature of said phenomenon: this 
es consecuencia del modelo androcéntrico socialmente aceptado en un entorno cultural donde la masculinidad, con todos sus modelos y formas de ejercer el poder, es lo hegemónicamente aceptado.

Se propone el término transmasculinización en el sentido de la manera cómo una estructura o modelo androcéntrico puede influir en las acciones de poder cuando este es ejercido por las mujeres. Este concepto busca definir un proceso mental a nivel individual que, cuando se expresa en lo social, hace que las mujeres, en la medida que ocupan cargos de poder, lo ejerzan adoptando formas y modelos masculinos para lograr ejercer de manera legítima dicho espacio de poder.

\section{Palabras clave}

Hegemonía - América Latina; Androcentrismo - América Latina; Desigualdad social - América Latina; Mujeres en la política - América Latina; Poder político - América Latina; Discriminación de género - Aspectos políticos - América Latina.

\section{Introducción}

Las reflexiones presentadas aquí son producto de una investigación exploratoria, en la cual se analizó la participación de mujeres en cargos de poder en diversos países de la región latinoamericana, y a través de una aproximación cualitativa se visualizó su participación en el sistema político al que representaban, definiendo sus modelos de ejercicio del poder y así determinar cómo se desarrolla el fenómeno de la transmasculinización.

Un ejemplo de ello puede observarse en la manera en como la primera y única vicepresidenta hasta la fecha en Guatemala, Roxana Baldetti, desarrolló su liderazgo, el cual no generó ningún impacto en el imaginario ni en la estructura sociopolítica del Estado guatemalteco. Esto a diferencia de Michelle Bachelet en Chile, quien no solo generó un cambio en el imaginario político, sino que también logró establecer de manera efectiva, dentro de la estructura del Estado, un Ministerio que visibiliza la necesidad de una visión inclusiva a todo nivel en la gestión pública. is a consequence of the socially accepted androcendric model in a cultural environment where masculinity, with all its models and forms to exert power, is hegemonically accepted.

The term transmasculinization has been proposed in the sense in which how an androcentric structure or model can exert an influence on the actions of power when this is practiced by women. This concept intends to define a mental process at the individual level that, when socially expressed, makes that women-to the extent to which they occupy positions of power-exercise it by adopting masculine forms to legitimately practice said space of power.

\section{Keywords}

Hegemony - Latin America; Androcentrism - Latin America; Social inequality - Latin America; Women in politics - Latin America; Political power - Latin America; Gender discrimination - Political aspects - Latin America.

Fuera de pocas excepciones, las mujeres en cargos de poder adoptan formas y modelos masculinos al ser este modelo socialmente aceptado en un entorno cultural donde la masculinidad, con todos sus modelos y formas de ejercer el poder, es hegemónico.

Hay una serie de relaciones de poder en las que las mujeres adoptan identidades autoconstitutivas del poder mismo, negando nuestra propia identidad. Esta idea de transmasculinización busca así conceptualizar el fenómeno al que se ven expuestas las mujeres que optan o asumen cargos de poder que tradicionalmente han sido ocupados y hegemonizados por hombres.

\section{Metodología}

A través de un análisis cualitativo del discurso, utilizando herramientas hemerográficas, se realizó una comparación entre el ejercicio del poder y los grados de transmasculinización que generan correlaciones positivas o negativas en las diferentes gestiones de las mujeres que han ocupado la presidencia de sus respectivos países en América Latina; y en segunda instancia, una comparación 
a través de la reconstrucción histórica para entender los límites y alcances que dicho proceso de ejercer el poder de manera androcéntrica pone a la lucha por una agenda política desde las mujeres en el mundo.

\section{Resultados y discusión}

El concepto de transmasculinización alude a una manera de ejercer el poder en medio de un modelo hegemónico con amplia predominancia masculina. Tanto hombres como mujeres se ven afectados al ejercer un cargo de poder en una estructura con bases androcéntricas, las cuales desde su origen determinan ciertas características o conductas masculinas predeterminadas o esperadas al momento que una persona ejerce o lidera cargos de poder.

Esta idea nos permite entender que, en donde existe una forma determinada de percibir y ejercer el poder, un actor de cierta relevancia, independientemente de su género, es presionado por un entorno dominante, el cual condiciona su actuar de una manera determinada ante la sociedad, principalmente en los puestos de decisión política.

Para profundizar en esta idea, la noción de hegemonía planteada por Gramsci nos permitirá entender el contexto de relaciones de dominación en donde acaece nuestro concepto.

Para Gramsci, la idea de hegemonía debe entenderse como una relación social constituida por dos dimensiones: una base material ligada a la posición de las clases en la estructura social, pero que se realiza en la superestructura a través de una concepción del mundo que encarna la visión compartida que refleja los intereses de la clase dominante de una manera universalizada $y$, por lo tanto, parte del acervo de la sociedad en su conjunto (Gramsci, 1984, p. 33), al tiempo que se plasma de formas diversas en:

a) el sentido común,

b) las prácticas cotidianas $y$,

c) un tipo particular de Estado.

Sin embargo, estas formas y modelos de ejercer el poder han estado supeditados al complejo proceso de masculinización, ya que las mujeres han estado ausentes en este proceso, y, por tanto, la dimensión femenina del ejercicio del poder es un elemento que recién en este momento comienza a visibilizarse, aunque marcado aún por la masculinización, que siempre ha preponderado, pues en la organización social se determinan ciertos papeles y estereotipos, los cuales, con el tiempo, se naturalizan y se transforman en algo "socialmente aceptado".

Por ello, históricamente, las posiciones de poder y decisión han sido consideradas como espacios exclusivos para hombres. Si bien han existido mujeres en funciones importantes de poder a través de la historia y en diversos escenarios, estas han sido obviadas o deslegitimadas, en su mayoría, sometidas a un escarnio público y social criticándose su gestión de una forma severa, algo muy distinto al parámetro a que se ha sometido a un hombre en igualdad de circunstancias. Veamos ahora, como ejemplo de lo analizado por Bernal, en el caso de la expresidenta de Costa Rica, Laura Chinchilla, quien en el 2015 hacía un Ilamado a medios de comunicación para que estos modificaran la imagen de mujeres líderes, ya que consideraba que a menudo se minimizaban sus logros, así como que se las sometía a rigurosos escrutinios por su condición de género y no por el resultado de su gestión en sí.

Chinchilla enfatizaba que hay "sesgos desde el punto de vista de cobertura mediática o de minimización que los medios de comunicación hacen del liderazgo femenino", además resaltó que, al optar las mujeres a un cargo de poder", se enfrentan a un juicio más riguroso y complejo de manejar", sumado a ello considera que las mujeres siguen siendo juzgadas por "patrones de asimilación" masculinos; $y$, al no encajar con ese modelo, llegan a ser discriminadas.

Pero ¿quésucedecuando se formaunamasa crítica oungrupo relevante de mujeres ejerciendo cargos de poder? Antes analicemos las reflexiones de Tickner en cuanto a la forma de percibir y ejercer el poder, desde el feminismo de punto de vista y el concepto de hegemonía que fue desarrollado por Gramsci, y cómo ambos - teoría y concepto- influyen en lo que llamamos la transmasculinización de la mujer en el ejercicio del poder Tickner contempla en su teoría que el cambio de la percepción de poder toma otro aspecto al ser las mujeres, como grupo, quienes ejercen puestos de poder. En otras palabras, en la medida que haya un mayor número de mujeres en el mundo ejerciendo cargos relevantes, hasta el punto de generar una masa crítica y no solo casos aislados, como ha ocurrido hasta ahora, tanto las críticas hacia ellas como la función en sí misma que será ejercida podrán ser analizadas con una visión más femenina del mundo, por tanto, su valoración podría llegar a ser distinta. 
Tickner visualiza que el ejercicio del poder a cargo de mujeres podría dar un giro positivo a las relaciones internacionales, ya que éstas en su condición de género perciben el poder de una manera distinta a como generalmente lo perciben los hombres, esto a razón de como ellas y ellos se han desarrollado a lo largo de la historia (Guillaume, 2011).

Se debe mencionar que Tickner considera que, a través de la historia, el punto de vista y el papel de la mujer en los diversos escenarios han sido silenciados sistemáticamente (Lozano Vásquéz, 2012, p. 146). El enfoque con perspectiva de género tiene la capacidad de brindar un complemento y una visión menos parcial que la que actualmente se maneja, principalmente en la teoría realista de las relaciones internacionales.

El feminismo considera el poder vinculado con la idea de "persuasión" porque las mujeres han tenido, en general, menos acceso a instrumentos coercitivos que los hombres, y por ello "el feminismo de punto de vista sostiene que existe un punto de vista específicamente femenino del mundo, distinto del que tienen los hombres, susceptible de cambiar las relaciones internacionales para hacerlas más cooperativas y pacíficas" (Guillaume, 2011, p. 15).

Lo anterior implica el comprender la percepción de poder y cómo las mujeres, a través de sus acciones como grupo, pueden generar cambios en los escenarios del sistema. En este aspecto, recogemos la idea expresada por Guillaume basándose en lo planteado por Tickner, en el sentido de que las mujeres que han alcanzado el poder lo han hecho de manera individual y haciendo prevalecer preferentemente habilidades duras, con el fin de ser reconocidas entre sus pares y no ser criticadas por ser blandas en el ejercicio del poder, cayendo de este modo en el proceso de transmasculinización.

En la medida en que no haya un número considerable de una visión femenina o mujeres líderes que presionen hacia un cambio cultural mayor en las relaciones internacionales y en las maneras de ejercer el poder, las mujeres seguirán expuestas a someterse a un proceso de transmasculinización y a las críticas constantes en un medio predominantemente masculinizado y machista que terminará buscando cualquier forma para desacreditar su gestión.

Vemos que, cuando las mujeres han intentado o han alcanzado cargos de poder en el ejecutivo en América Latina, cada una de ellas ha ocupado una posición donde ha sido criticada, si bien ellas trazaron un quiebre en el imaginario social de forma positiva para otras mujeres y la sociedad en general, demostrando que las mujeres pueden optar por y ejercer cargos de poder. Es necesario que las mujeres desarrollen una conciencia de su condición de género para poder plantear cambios en la estructura del Estado. Examinemos el caso de la expresidenta de Chile, Michelle Bachelet, quien fue la primera presidenta electa en dos períodos no consecutivos y que tuvo ese enfoque de género durante su gestión, implementando así un cambio en la estructura del Estado al instaurar el Ministerio de la Mujer y Equidad de Género.

Se puede decir que la visión de género no se posee solo por ser mujer, se adquiere al realizar una reflexión de las desigualdades y las condiciones diferenciadas de desarrollo en la sociedad entre hombres y mujeres.

Las mujeres en altos cargos que no presentan esta visión, de forma consciente, de su condición de género, reciben críticas sociales, las cuales hacen visible la importancia de que ellas asuman responsabilidades y compromisos con sus pares mujeres en la estructura social a través de una gestión más inclusiva.

Además, todas las estructuras del Estado están configuradas de acuerdo con una cultura machista predominante donde el hombre tiene todo el marco jurídico y estructural para ejercer el poder. La mujer, por el contrario, en la medida que escala posiciones de poder, se encuentra con una serie de obstáculos, muchas veces insalvables, para poder continuar su ascenso; es lo que algunos han definido como el techo de vidrio que deben sortear.

Un ejemplo de lo anterior es la creación de instituciones específicas para las primeras damas o esposas de los presidentes, que en la mayoría de los casos cumplen funciones secundarias, solo de acompañamiento al mandatario o de acción social, sin ninguna incidencia en los asuntos del Estado, y que por su estructura solo están destinadas a ser usadas por mujeres como "las esposas de...".

Las mujeres que han optado por la participación política, por una carrera en la esfera pública y por cargos no tradicionales acordes con su papel societal, por su condición de género, han sido fuertemente criticadas y observadas por distintos sectores de la población que aún no asimilan a una mujer en cargos de poder por el enraizamiento, muchas veces de manera inconsciente, de una estructura patriarcal como una conducta o pensamiento machista. 
Volvemos a recalcar que aún no se ha generado un grupo considerable de mujeres que gobiernen, con el fin de establecer una masa crítica que permita el uso mayoritario de poder blando en el ejercicio del poder, y que, a su vez, no se vean expuestas a una crítica desde el punto de vista masculino, siendo estas sexualizadas, discriminadas 0 invisibilizadas por un tema de género ante una sociedad y estructura política fuertemente machista, sexista y parcializada.

Dilma Rousseff atribuyó al machismo la imagen de "dura" que le han adjudicado. Dice: "De la mujer se espera una fragilidad. Por lo menos, la imagen que se tiene es que la mujer es frágil (...) y cuando una mujer asume una posición de mando, de autoridad, es vista como fuera de su papel" en la medida en que la tendencia sea a una mayor participación femenina, se dará un proceso inverso respecto a la transmasculinización, esto porque históricamente los modelos de construcción social del poder siempre, o en su mayoría, han sido desarrollados y ejercidos por el género masculino. Por lo tanto, las mujeres, al optar por o alcanzar cargos de poder no tradicionales, no cuentan con modelos distintos a los ya desarrollados o preestablecidos por el género masculino en la estructura social, lo que conlleva, de forma consciente o inconsciente, la necesidad de adoptar estos modelos preestablecidos tanto en su ascenso como en el ejercicio mismo del poder.

Sin embargo, pueda que, en la medida en que haya una mayor participación del género femenino en los cargos de poder a escala internacional y nacional, estos modelos y parámetros deban irse modificando y adaptarse a las nuevas formas del ejercicio del poder.

Por tanto, a una mayor participación del género femenino, las formas de ejercicio del poder propias de su género se pueden ir desarrollando, adoptando y ejerciendo ante la nueva realidad existente, esto incluye una visión más amplia de la gestión pública, partiendo de la importancia de su condición de género. Por lo anterior, no sería extraño que en un futuro nos encontremos con representantes del género femenino ejerciendo el poder a escala internacional con rasgos y modelos propios que los caractericen.

En el camino que la mujeres deben recorrer para tener una mayor participación política, la hegemonía cultural, las estructuras de un Estado machista y los propios rasgos atávicos que se arrastran en medio de un escenario adverso las obliga, de una forma consciente o inconsciente, a asumir actitudes que no son propias de su género o de sus formas

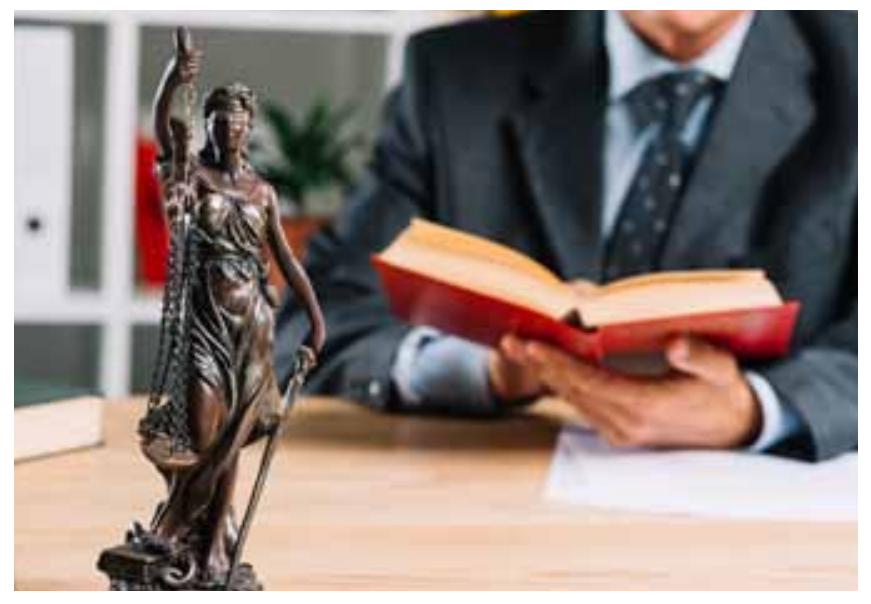

de ejercer el poder, ya que en la mayoría de los casos, en cuanto se suscitan dudas respecto de sus decisiones, se culpa de manera descarnada a su condición de género y a lo que su género representa ante una estructura patriarcal y machista que aún no visualiza a una mujer en cargos o papeles no tradicionales en la sociedad. A lo señalado anteriormente, la historia muestra que las mujeres, para llegar al poder, en la mayoría de los casos, en el camino han debido adoptar costumbres, formas y modelos de ejercicio del poder que no siempre han sido acordes con su género, pasando por un proceso de transformación y adoptando las formas y costumbres masculinas, más acordes con las estructuras machistas preponderantes.

Ese proceso de transformación es lo que en este estudio se ha definido como transmasculinización en el ejercicio del poder. Un ejemplo clásico de este proceso lo podemos encontrar en el ascenso al poder de Margaret Thatcher, exprimera ministra del Reino Unido (1979-1990). Si Thatcher no hubiese resaltado esa característica de líder "dura", muy asociada con la masculinidad, difícilmente hubiese llegado a ser primera ministra. Se debe resaltar que la estructura sociopolítica está predeterminada a exigir una postura "dura" del líder a cargo.

Las mujeres que transitaron como Thatcher para alcanzar el poder, en el mundo contemporáneo, han debido adaptarse a estas condiciones, y de una manera u otra se han visto obligadas a adoptar formas de ejercer el mando acordes con la cultura hegemónicamente existente, razón por la cual se puede llegar a anular su propia concepción del poder, o del ejercicio del poder, desde su condición de género, y como consecuencia adoptar, total o parcialmente, aquella que ha existido siempre caracterizada por una visión masculina. 
Se puede señalar que las mujeres que han alcanzado el poder, a través de sus acciones, han sido conscientes de lo difícil que son los escenarios que se les presentan y de los obstáculos que han debido superar para lograr sus objetivos y gestionarlos de manera eficiente, razón que las han llevado a adoptar determinadas maneras y formas de ejercerlo, pero la adopción de las formas, conductas y modelos masculinos se hace mayoritariamente de manera inconsciente, ya que en el imaginario social el modelo preponderante de ejercer ese poder sigue siendo el desarrollado por los hombres y es el que ha sido aceptado social y culturalmente, por tanto, se convierte en su modelo conductor.

Lo anterior lleva a que la transmasculinización, en el ejercicio del poder, sea un proceso inconsciente para la mayoría de las mujeres que han ejercido cargos de poder, aunque de momento se considera que la experiencia empírica no permite efectuar una validación completa del postulado planteado por Ann Ticknner, que dice que con un mayor grupo de mujeres en cargos de poder habría un cambio sustancial en las relaciones internacionales, ya que solo una única vez se ha dado el caso mencionado en América Latina; y el período de coincidencia entre las mandatarias fue muy breve como para establecer la validez de esa afirmación y de esta manera anular el proceso de transmasculinización.

\section{Conclusiones}

Aunque de momento se considera que la experiencia empírica no permite efectuar una validación por completo del postulado planteado por Ann Ticknner, que dice que con un mayor grupo de mujeres en cargos de poder habría un cambio sustancial en las relaciones internacionales, ya que solo una única vez se ha dado el caso mencionado en América Latina y el período de coincidencia entre las mandatarias fue muy breve como para establecer la validez de esa afirmación y de esta manera anular el proceso de transmasculinización.

La comprensión del patriarcado debe realizarse en términos de las relaciones entre sujetos y no como meros actores sustantivos, por lo que el énfasis de esta propuesta es cuestionar la naturalización de lo que a toda luz es un hecho histórico y relacional, y por ende posible de ser disputado políticamente. Las relaciones de poder son creadoras de identidades y los órdenes de opresión generan sus propias vías y tránsitos donde ese poder es posible de ser ejercido a través de la identidad como un acto relacional. Eso hace vital, en conclusión, el cuestionarse el quiénes somos y por qué podemos hacer lo que hacemos en virtud de lo que creemos ser en los puestos de decisión política, en los dispositivos de control social, etc. Por ello, es que no se trata únicamente de desplazar a los titulares tradicionales del poder, es decir, a los hombres, sino de cuestionar la constitución y naturaleza del poder mismo.

Además de la necesaria superación de estos papeles y estereotipos sociales que influyen en el ejercicio del poder, principalmente hacia las mujeres, que anulan o desvirtúan la capacidad de ejercer el poder desde una visión feminista del mundo donde las mujeres en el poder pueden influir hacia un orden cooperativo y más incluyente.

\section{Referencias}

Arenal, C. (2010). Introducción a las Relaciones Internacionales ( $4^{\mathrm{a}}$ ed.). Madrid, España: Tecnos.

Arendt, H. (2016). La condición humana [versión de Adobe Acrobat Reader]. Recuperado de https://www. planetadelibros.com/libros_contenido_extra/32/31441_ La_condicion_humana.pdf

Gramsci, A. (1980). Notas sobre Maquiavelo: sobre la política y el Estado moderno. [versión de Adobe Acrobat Reader]. Recuperado de https://kmarx.files.wordpress. com/2012/04/gramsci-notas-sobre-maquiavelopolc3adtica-y-estado-moderno.pdf

Gramsci, A. (2015). Hegemonía y lucha política en Gramsci. Buenos Aires, Argentina: Ediciones Luxemburgo.

Guillaume, E. (enero, 2011). El feminismo de punto de vista como teoría de las relaciones internacionales. Trabajos y Ensayos, (13), 1-18. Recuperado de http://www.diprriihd. ehu.es/revistadoctorado/n13/Estelle_Guillaume13.pdf

La Gaceta. (04 de febrero de 2006). Isabel Perón, la primera mujer que ejerció la presidencia. La Gaceta. Recuperado de http://www.lagaceta.com.ar/nota/145747/argentina/ isabel-\%20peron-primera-mujer-ejercio-presidencia. html

Londoño, E. (24 de julio de 2017). Michelle Bachelet y el eclipse de las mujeres en la política de América Latina. The New York Times. Recuperado de https://www. nytimes.com/es/2017/07/24/espanol/america-latina/ michelle-bachelet-mujeres-politica.html 
Lozano Vásquéz, A. (septiembre-diciembre, 2012). El Feminismo En las Relaciones Internacionales: Un breve repaso. Revista de Relaciones Internacionales de la UNAM, (114), 143-152.

Morello, A. (23 de agosto de 2017). Dura crítica de Isabel Allende: Las mujeres tienen que ganar su espacio a patadas. FiloNews. Recuperado de https://www.filo. news/actualidad/A-quienes-no-podran-cortarle-losservicios-esenciales-20200326-0044.html

NTX. (03 octubre del 2015). Las mujeres podemos vencer cualquier obstáculo: Chinchilla. Informador. MX. Recuperado de https://www.informador.mx/ Suplementos/Las-mujeres-podemos-vencer-cualquier-
obstaculo-Chinchilla-20151003-0122.html

Nye, J. S. (2011). Las cualidades del líder. Barcelona: Ediciones Paidós.

Padilla, L. A. (2009). Paz y conflicto en el siglo XXI: teoría de las relaciones internacionales (2a ed.). Guatemala: IRIPAZ.

Villaroel Peña, Y. U. (julio-diciembre, 2007). Los aportes de las teorías feministas a la comprensión de las relaciones internacionales. Revista Politeia, 30(39), 65-86. Recuperado de https://www.redalyc.org/ pdf/1700/170018341003.pdf 